\title{
Factors Associated with Low Immunization Coverage in Children Under Five Years in Asuogyaman District in Eastern Region of Ghana
}

\author{
Solomon Antwi Brefo ${ }^{1 *} \quad$ Abdul Aziz Abdulai ${ }^{2} \quad$ Cyril Azornu Kwami $^{1} \quad$ Jonathan Mawutor Gmanyami $^{1}$ \\ Emmanuel Senyo Kasu ${ }^{1,3}$ \\ 1.School of Public Health, University of Health and Allied Sciences, Ho, Volta Region, Ghana \\ 2.District Health Directorate, Ghana Health Service, Asuogyaman District, Eastern Region \\ 3.Public Health Department, Ho Teaching Hospital, Ho, Volta Region
}

\begin{abstract}
Expanded Programme on Immunization is one of the most successful and cost effective programmes adopted by World Health Organization and other partners to prevent vaccine preventable diseases which are the major cause of infant morbidity and mortality worldwide. Since 2012, Africa has demonstrated unprecedented commitments in the Global Vaccine Action Plan and this has resulted in the protection of millions of children. Despite this, some infants still remained unvaccinated and at risk of vaccine preventable diseases. Even though, Ghana was among countries which achieved 95\% DPT3 coverage, this was not evenly distributed across all districts and Asuogyaman District was not an exception. The study assessed the factors associated with low immunization coverage among children under five years in Asuogyaman District. A descriptive cross-sectional study was conducted among mothers/caregivers in the Asuogyaman district. A total of $401 \mathrm{mothers} /$ caregivers were enrolled into the study and structured questionnaire was used to collected data from mothers/caregivers on factors associated with low immunization coverage in children under 5 years. The caregivers were mostly females, 396(98.7\%) with mean age of 28.6( \pm 6.7$)$ years and the majority of them, $216(53.9 \%)$ were in the age group 20-29 years. Only 250(62.3\%) of the participants had good knowledge on immunization. The level of knowledge of the caregivers was significantly associated with ethnicity $\left(\chi^{2}=9.83, \mathrm{p}=0.043, \alpha=0.05\right)$, occupation $\left(\chi^{2}=10.92, p=0.012, \alpha=0.05\right)$, and place of delivery $\left(\chi^{2}=4.37, p=0.037, \alpha=0.05\right)$. Participants who were selfemployed were $38 \%$ more likely to have good knowledge compared to those who were unemployed $(\mathrm{AOR}=0.62$; $95 \% \mathrm{CI}=0.40-0.95 ; \mathrm{p}=0.030)$ and those who delivered at home were also twice more likely to have good knowledge on immunization compared to those who delivered at the health facility (AOR $=2.12 ; 95 \% \mathrm{CI}=1.19$ $3.77 ; \mathrm{p}=0.010)$. Most of the caregivers, 304(75.8\%) had positive attitude and good practices, $346(86.3 \%)$ on immunization. Majority of the mothers/caregivers, 353(88.0\%) perceive health workers had positive attitude towards them.
\end{abstract}

Conclusion: Immunization services were largely accessible to the caregivers. Knowledge of mothers/caregivers on immunization was low, but they had positive attitude and good practices on immunization.

Keywords: Immunization, coverage, Asuogyaman district, Eastern region, Ghana

DOI: $10.7176 / \mathrm{JHMN} / 81-09$

Publication date:October $31^{\text {st }} 2020$

\section{INTROUCTION}

According to World Health Organization "Immunization is the process whereby a person is made immune or resistant to an infectious disease, typically by the administration of a vaccine. Vaccines stimulate the body's own immune system to protect the person against subsequent infection or disease"(WHO, 2015). Immunization is safe, cost effective and prevents morbidity, disability and mortality from vaccine-preventable diseases including polio, cervical cancer, rotavirus diarrhea, diphtheria, hepatitis B, measles, mumps, pertussis (whooping cough), pneumonia, rubella, meningitis and tetanus (WHO, 2018). It is also very effective in children by producing antibodies to protect children from diseases which improves their health and development.

The global effort to use vaccination as a public health intervention began when the World Health Organization launched the Expanded Programme on Immunization (EPI) in 1974. Globally 2 to 3 million deaths due to diphtheria, pertussis (whooping cough), tetanus, and measles can be prevented by immunization and an extra 1.5 million deaths could be avoided if global vaccination coverage improves (WHO, 2017).

In 2012, the Global Vaccine Action Plan (GVAP) was signed by 194 WHO member states, who pledged to ensure that no person misses out on vital immunization with a target of 90\% DTP3 immunization coverage in all countries by the year 2015 (WHO/UNICEF, 2015). By 2016, about 86\% of children worldwide (116.5 million infants) received 3 doses of Diphtheria-Pertussis-Tetanus (DPT3) vaccine with 130 countries reaching at least 90\% DPT3 vaccine coverage (WHO, 2017).

In Africa, although the immunization coverage has been on a steady rise from $57 \%$ in 2000 to $76 \%$ in 2015 for routine coverage for Diphtheria-Pertussis-Tetanus (DPT) as a proxy, the coverage is low as compared to 
global Diphtheria-Pertussis-Tetanus (DPT) immunization coverage of 86\% (Mihigo R, Okeibunor J\&, Anya B, Mkanda P, 2017). Since African agreed to the ambitious and unprecedented commitments in the Global Vaccine Action Plan (GVAP) in 2012, vaccination has protected millions of children in African (WHO, 2016). However, in 2014, the number of infants who did not receive the third dose of DPT vaccines in the WHO African Region was estimated to be 7.4 million out of an annual birth cohort of 32.7 million; (Mihigo R, Okeibunor J\&, Anya B, Mkanda P, 2017).

In Ghana, the EPI Programme was established in June 1978 with six (6) antigens consisting of BCG, measles, Diphtheria-Pertussis-Tetanus (DPT) and oral polio vaccine for infants. This intervention has reduced morbidity, disability and mortality, in Ghana (Ministry of health, 2016). Tetanus toxoid (TT) vaccination was also introduced to prevent tetanus in pregnant women/mother and neonates. The introduction of the immunization programme was in response to the National Health Policy to reduce illness and deaths due to vaccine preventable diseases (VPDs) contributing massively to the reduction of both infant and child mortality. The EPI programme over the years has been expanded to include other vaccines leading to the introduction of Yellow fever in 1992 and in 2002, Hepatitis B and Haemophilus influenza type B (Hib) was added to DPT to become Pentavalent (Ministry of health, 2016). In 2012, The Ministry of Health introduced two additional vaccines, the pneumococcal, rotavirus and measles second dose at 18 months. In 2013, measles vaccine was replaced with a rubella-containing measles vaccine (Measles-Rubella (MR) vaccine) and in 2016, Meningitis A was also introduced.

Ghana Health Service annual report 2016 indicates a high immunization coverage for all the antigens using PENTA 3 as proxy which was 99\%. However, vaccination coverage in Asuogyaman District was lower than the national coverage. Data obtained from Asuogyaman District Health Directorate (DHD) annual report 2017 indicates a tremendous decrease in the trend of immunization coverage for all the antigens from 2015 to 2017(as shown in figure 1 and 2) (Asuogyaman DHD report, 2017). This low immu nization coverage has resulted in occurrence of confirmed Vaccine Preventable Diseases (VPDs) such as measles (2) and meningitis (1) in the district (Asuogyaman DHD report, 2017). Although there has been a lot of activities to improve the immunization coverage such as creating of new outreach points, increase in home visit and introduction of school immunization, the coverage is still not improving. The persistence decrease in coverage can be attributed to so many factors associated with low coverage. The study assessed the factors associated with low immunization coverage in children under five years in Asuogyaman district.

\section{Three year trend of immunization - Asuogyaman District}

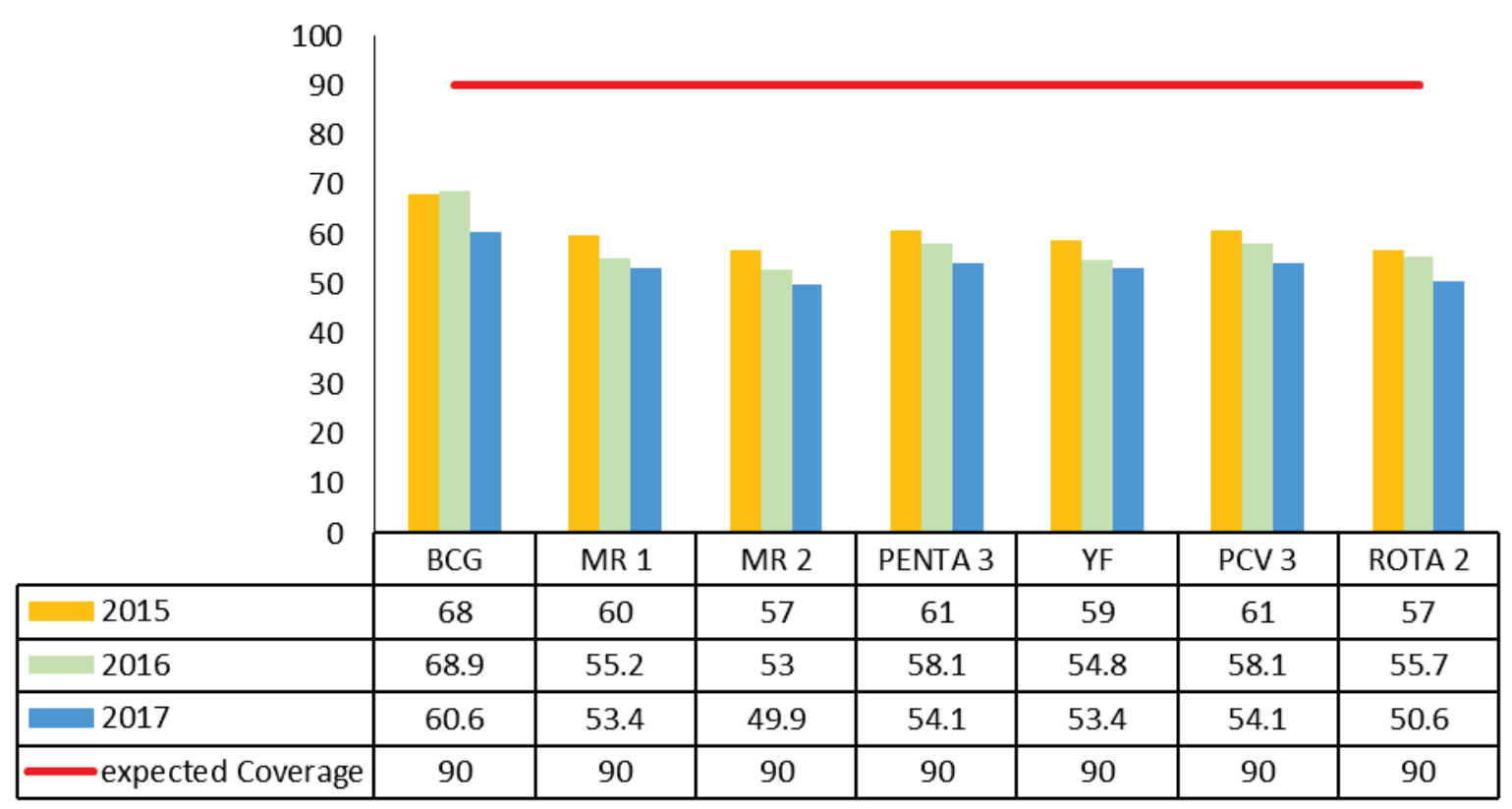

Figure 1: EPI coverage of Asuogyaman District (2015-2017) 


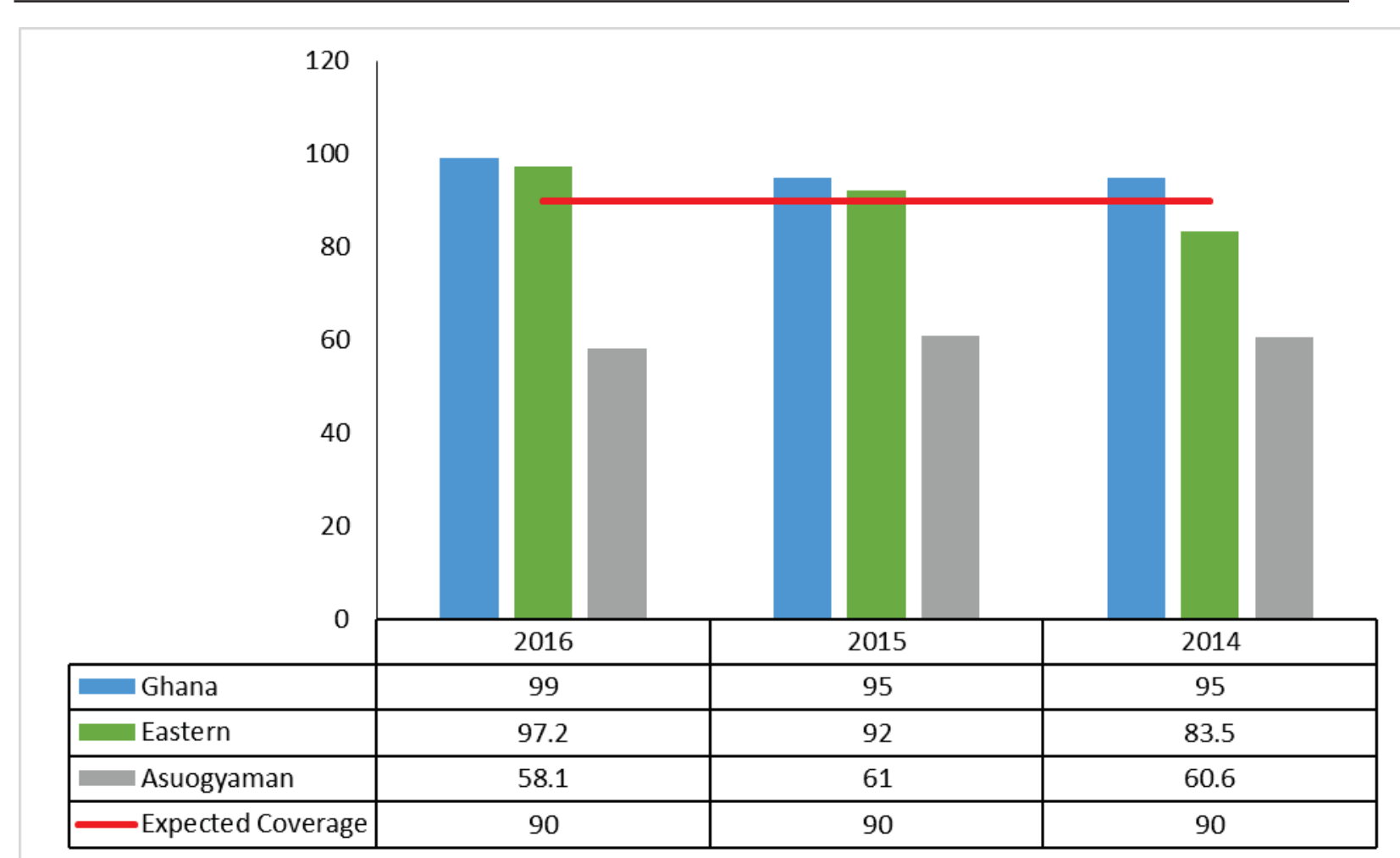

Figure 2: 2016 PENTA 3 coverage for Ghana, Eastern Region and Asuogyaman District Compared

\section{STUDY METHODS}

\section{Study design}

A descriptive cross-sectional study was conducted and structured questionnaires were used to collect the data from the participants. Ethical approval was obtained from the University of Health and Allied Sciences Ethics Review Committee (UHAS-ERC) with reference number UHAS-REC A4 [95] 18-19.

\section{Study setting and population}

Asuogyaman District is one of the twenty six districts in the Eastern Region of Ghana. It was created in 1988, covering a total estimated surface area of 1,507 square kilometers with its capital at Atimpoku (Asuogyaman district profile, 2017). The district shares boundary with Afram Plains District in the North, North Tongu District in the South, Manya Krobo District in the West and South Dayi District in the East (Asuogyaman district profile, 2017).

Asuogyaman District has about 122 communities divided administratively into six (6) sub-districts namely: Akosombo, Atimpoku, Senchi, Akwamufie-Apeguso, Anum-Boso and Adjena-Gyakiti.

Akosombo has one CHPS zone, Atimpoku, Senchi, Anum-Boso and Ajena-Gyakiti have 5 CHPS zones each and also Akwaufie-Apegusu has 7 CHPS zones (Asuogyaman district profile, 2017).

Asuogyaman district has a projected population of 107,166 out of which 21,258 ( $20 \%$ of total population) are children under 5.

Health Facilities and number

\begin{tabular}{|l|l|}
\hline TYPE OF FACILITY & NUMBER \\
Hospital & 1 \\
Health centers & 10 \\
Mission clinic & 1 \\
Private clinics & 2 \\
Outreach centers & 79 \\
CHPS & 28 functional \\
\hline
\end{tabular}

\section{Data collection and sampling}

Data collection was carried out by trained research assistants using pre-tested structured questionnaires on demographics characteristics of study participants and also on questions related to factors associated with low immunization coverage of children under five years in the district.

Data was collected at the household level in each of the selected communities which were identified by 
special codes. An estimated 15-20 minutes was spent with each respondent.

Data were collected from selected communities from all the six (6) sub districts in the district from caregivers of children under five (5) years. A proportionate sampling technique was used to select the participants from each sub district. The proportion of children under five years in each subdistrict was determined and used to estimate for the participants from each sub district.

The sub district populations for under five years used for the calculation of the sample size were as follows;

\begin{tabular}{lll}
\hline S/N & Sub-District & Population \\
\hline 1 & Akosombo & 3401 \\
2 & Atimpoku & 2551 \\
3 & Senchi & 2551 \\
4 & Anum/Boso & 4464 \\
5 & Akwamufie & 5102 \\
6 & Adjena & 3189 \\
Total & & 21258 \\
\hline
\end{tabular}

\section{Sample Size Determination}

The sample size was calculated based on the district's Penta 3 immunization coverage for 2017 which was $54.1 \%$ which served as a proxy for immunization coverage (Asuogyaman District Health Directorate, 2017), margin of error of 0.05 and $95 \%$ confidence level.

Sample Size Estimation

$$
\begin{aligned}
& \mathrm{n}=\frac{\left(Z_{\alpha / 2}\right)^{2} p q}{d^{2}} \\
& \text { Where } \mathrm{n}=\text { sample size required } \\
& \mathrm{Z}=\mathrm{Z} \text { score } \\
& \mathrm{p}=\text { coverage } \\
& \mathrm{q}=1-\mathrm{p} \\
& \mathrm{d}=\text { margin of error }
\end{aligned}
$$

Where $\mathrm{z}=1.96$ at $95 \%$ confidence level, $\mathrm{p}=54.1 \%$ or $0.541, \mathrm{q}=1-0.541=0.459$ and $\mathrm{d}=0.05$

$$
n=\frac{1.96^{2} * 0.541(0.459)}{0.05^{2}} \quad \begin{aligned}
& n=\frac{3.8416 * 0.2483}{0.0025} \\
& \mathrm{n}=381.56=382
\end{aligned}
$$

Adding 5\% non-respondent rate
$\mathrm{n}=382 * 0.05=19.1=19$
Total sample size $=19+382=\mathbf{4 0 1}$

Hence the sample size for Akosombo sub district was

$\frac{3401}{2.12 .58} \times 100=16.0 \%$

Hence the actual sample size for Akosombo $=\frac{16}{100} \times 401=64$

The sample size for Atimpoku sub district was

$\frac{2551}{2.12 .58} \times 100=12.0 \%$

Hence the actual sample size for Atimpoku $=\frac{12}{100} \times 401=48$

The sample size for Senchi sub district was

$\frac{2551}{2.12 .58} \times 100=12.0 \%$

Hence the actual sample size for Senchi $=\frac{12}{100} \times 401=48$

The sample size for Anum/Boso sub district was

$\frac{4464}{21258} \times 100=21.0 \%$

Hence the actual sample size for Anum $/$ Boso $=\frac{21}{100} \times 401=84$

The sample size for Akwamufie sub district is

$\frac{5102}{2.12 .58} \times 100=24.0 \%$

Hence the actual sample size for Akwamufie $=\frac{24}{100} \times 401=96$

The sample size for Adjena sub district was

$\frac{3189}{2.12 .58} \times 100=15.0 \%$

Hence the actual sample size for Adjena $=\frac{12}{100} \times 401=60$

From the calculation, 64 participants were selected from Akosombo sub district, 48 participants from 
Atimpoku sub district, 48 participants from Senchi sub district, 84 from Anum/Boso sub district, Akwamufie sub district were 96 and Adjena sub district were 60 participants respectively.

Simple random sampling method was used to select 4 communities from each sub district from a sampling frame consisting of the list of the communities in each sub district by lottery method. The total participants from each sub district were divided by four (4) to get the number of participants for each selected community. Purposive sampling method was used to select the mothers/caregivers interviewed from each house holds from the selected communities.

\section{Data analysis}

EpiData manager version 4.0.2.101 was used to create the database template and exported to EpiData entry version 4.0.2.49 for the data entry. After the entry, it was exported to excel for data cleaning and then to Stata version 13 for data analysis.

Microsoft Excel version 2013 was used to draw graphs. Two approaches for data analysis that is descriptive and analytic were used. The descriptive approach included calculation of the frequencies, percentages, tables, charts and graphs whiles the analytic approach included cross tabulations was used to calculate the associations between the variables under study and the significant levels with the use of chi-square.

RESULTS

Table 1: Demographic Characteristics of participants

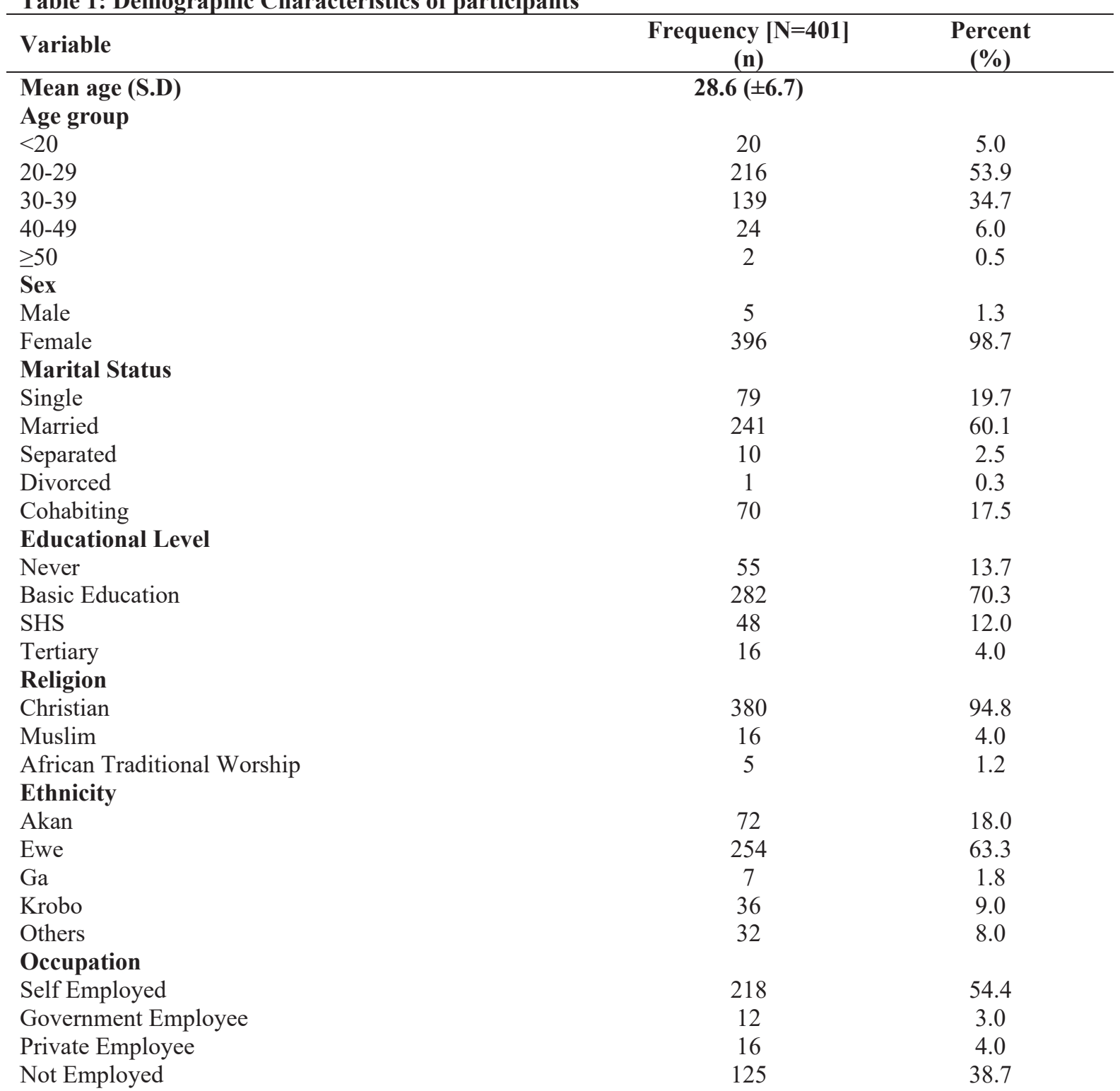




\begin{tabular}{lcc}
\hline Variable & $\begin{array}{c}\text { Frequency [N=401] } \\
\text { (n) }\end{array}$ & $\begin{array}{c}\text { Percent } \\
(\%)\end{array}$ \\
\hline Income (GHC) & & 58.9 \\
$<200$ & 123 & 24.9 \\
$200-400$ & 52 & 7.7 \\
$401-600$ & 16 & 4.3 \\
$601-800$ & 9 & 4.3 \\
$>800$ & 9 & 9 \\
\hline
\end{tabular}

Table 1 shows the demographic characteristics of the study participants. A total of 401 mothers/caregivers were enrolled in the study. The mean age of the study participants was $28.6 \pm 6.7$ years with most of the caregivers in the age groups 20-29 years (53.9\%) and 30-39 years $(34.7 \%)$. Only $2(0.5 \%)$ of the mothers/caregivers were teenagers and 44(9.0\%) were 40 years and older. Almost all the caregivers were females, 396(98.7\%). Most of the participants, 241(60.1\%) were married and only $11(2.8 \%)$ were separated or divorced. Majority of the participants, $346(86.3 \%)$ had some level of formal education consisting of primary/junior high, 282(70.3\%), senior high, 48(12.0\%) and tertiary, 16(4.0\%). A majority of the participants were Christians, 380(94.8\%) with the rest being Muslims and African Traditionalist. Most of the participants were Ewes 254(63.3\%) and only 72(18.0\%) were Akans. In terms of employment, 61.3\% of the participants were employed, whilst as much as $125(38.7 \%)$ were unemployed. Those who were employed were selfemployed (54.4\%), government employees $(3.0 \%)$ and private employees $(4.0 \%)$. Majority of those who were employed had an income level below 200 Ghana Cedis (58.9\%) and only 9(4.3\%) had an income level above 800 Ghana Cedis per month.

Table 2: Characteristics of Children of Mothers/Caregivers attending Immunization Sessions

\begin{tabular}{lcc}
\hline Variable & $\begin{array}{c}\text { Frequency [N=401] } \\
\text { (n) }\end{array}$ & $\begin{array}{c}\text { Percent } \\
\text { (\%) }\end{array}$ \\
\hline Age of Child (Months) & & 22.4 \\
$0-11$ & 90 & 29.9 \\
$12-23$ & 120 & 47.6 \\
$24-59$ & 191 & \\
Sex of Child & 171 & 42.6 \\
Male & 230 & 57.4 \\
Female & & \\
Place of Delivery & 327 & 81.5 \\
Health Facility & 74 & 18.5 \\
Home
\end{tabular}

Table 2 shows the characteristics of children of mothers/caregivers attending immunization sessions. More than half of the children were females, $230(57.4 \%)$ and the majority of the children were in the age group of 2459 months, 191(47.6\%). Large proportion of the children 327 (81.5\%) were delivered in health facilities.

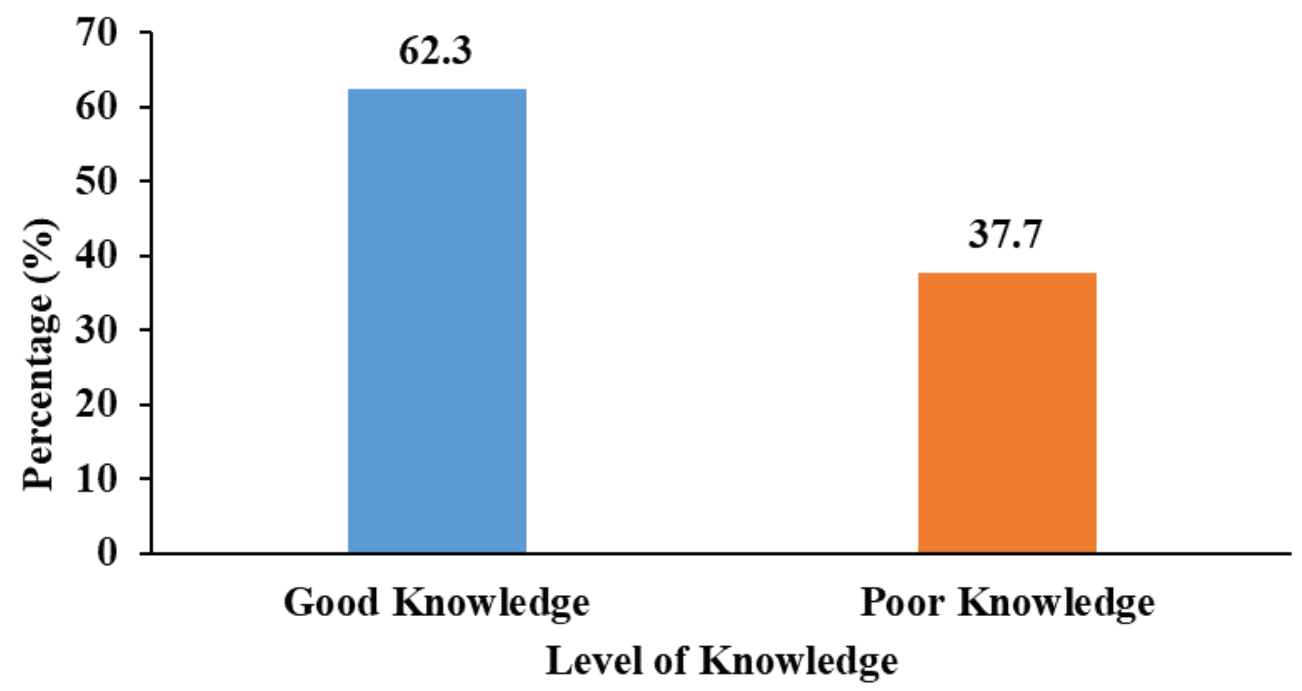

Figure 3 Level of Knowledge of Mothers/Caregivers on Immunization

Figure 3 shows the knowledge level of the mothers/caregivers on immunization. Overall, 62.3\% (250) of the caregivers had good knowledge while 151(37.7\%) had poor knowledge on immunization. 
Table 3: Knowledge of Mothers/Caregivers on Immunization

\begin{tabular}{|c|c|c|}
\hline Variable & $\begin{array}{l}\text { Frequency } \\
\text { (n) }\end{array}$ & $\begin{array}{l}\text { Percent } \\
(\%)\end{array}$ \\
\hline \multicolumn{3}{|c|}{ Heard about vaccination and vaccine preventable diseases } \\
\hline Yes & 394 & 98.2 \\
\hline No & 7 & 1.8 \\
\hline \multicolumn{3}{|c|}{ Source of information } \\
\hline Friends & 13 & 3.3 \\
\hline Relatives & 10 & 2.5 \\
\hline Television & 12 & 3.1 \\
\hline Health Worker & 339 & 86.0 \\
\hline Others & 20 & 5.1 \\
\hline \multicolumn{3}{|c|}{ Objective of vaccination } \\
\hline To prevent disease & 302 & 75.3 \\
\hline For healthy child & 70 & 17.5 \\
\hline It has no benefit & 1 & 0.3 \\
\hline Do not know & 28 & 7.0 \\
\hline \multicolumn{3}{|c|}{ Number of Vaccine-preventable Diseases known } \\
\hline One & 60 & 15.0 \\
\hline Two & 102 & 25.4 \\
\hline Three & 80 & 20.0 \\
\hline Four & 42 & 10.5 \\
\hline More Than Four & 36 & 9.0 \\
\hline I Don’t Know any & 81 & 20.2 \\
\hline \multicolumn{3}{|c|}{ Age at which child start routine EPI service } \\
\hline Just After Birth & 163 & 40.7 \\
\hline Weeks After Birth & 216 & 53.9 \\
\hline After One Year & 1 & 0.3 \\
\hline Anytime & 3 & 0.8 \\
\hline I Don’t Know & 18 & 4.5 \\
\hline \multicolumn{3}{|c|}{$\begin{array}{l}\text { Know the number of sessions required to complete routine EPI } \\
\text { services }\end{array}$} \\
\hline Yes & 221 & 55.1 \\
\hline No & 180 & 44.9 \\
\hline \multicolumn{3}{|c|}{ Number of sessions required } \\
\hline 1-2 Sessions & 7 & 3.1 \\
\hline 3-4 Sessions & 39 & 17.4 \\
\hline 5-6 Sessions & 69 & 30.8 \\
\hline 7 or more & 109 & 48.7 \\
\hline \multicolumn{3}{|c|}{ Age at which a child completes routine EPI services } \\
\hline 6-8 Months & 2 & 0.5 \\
\hline 9 Months & 71 & 17.7 \\
\hline 18 Months & 328 & 81.8 \\
\hline \multicolumn{3}{|c|}{ Know about the contra-indications of vaccine } \\
\hline Yes & 180 & 44.9 \\
\hline No & 221 & 55.1 \\
\hline \multicolumn{3}{|c|}{ Contra-indications to immunization } \\
\hline Fever & 51 & 26.6 \\
\hline Swelling & 63 & 32.8 \\
\hline Rash & 13 & 6.8 \\
\hline Convulsion & 3 & 1.6 \\
\hline Excessive Crying & 35 & 18.2 \\
\hline No side effect & 27 & 14.1 \\
\hline \multicolumn{3}{|c|}{$\begin{array}{l}\text { From table } 3 \text {, a total of } 394(98.2 \%) \text { had heard about vaccine preventable diseases, } 339(86.0 \%) \text { heard it } \\
\text { through health workers, and } 302(75.3 \%) \text { knew the objective of immunization was to prevent disease. Only } \\
36(9.0 \%) \text { of the participants knew more than four vaccine-preventable diseases while } 81(20.2 \%) \text { did not know } \\
\text { any vaccine-preventable disease. Also, only } 163(40.7 \%) \text { of the participants knew routine EPI services start at } \\
\text { birth while a majority } 216(53.9 \%) \text { thought it started } 6 \text { weeks after birth, and } 18(4.5 \%) \text { did not know the age at } \\
\text { which it starts. Most of the participants } 221(55.1 \%) \text { said they knew the number of sessions required to complete }\end{array}$} \\
\hline
\end{tabular}


routine EPI services, and 109(48.7\%) of them knew 7 or more sessions were required to complete the routine EPI services. Majority of the participants, 328(81.8\%) knew a child completes routine EPI services at 18 months and $180(44.9 \%)$ reported knowing about some contra-indications of vaccines. Among those who knew some contra-indications of vaccines, 51(26.6\%), 63(32.8\%), 13(6.8\%), 3(1.6\%), and 35(18.2\%) reported knowing fever, swelling, rash, convulsion, and excessive crying respectively as some contra-indications to vaccine

Table 4: Association Between Level of Knowledge of Mothers/Caregivers on Immunization and Demographic Characteristics

\begin{tabular}{|c|c|c|c|c|}
\hline \multirow[b]{2}{*}{ Variable } & \multicolumn{2}{|c|}{$\begin{array}{c}\text { Level of Knowledge } \\
\end{array}$} & \multirow[b]{2}{*}{$\begin{array}{c}\text { Chi-square } \\
\qquad \chi^{2}\end{array}$} & \multirow[b]{2}{*}{ p-value } \\
\hline & $\begin{array}{c}\text { Poor Knowledge } \\
{[\mathbf{N}=151]} \\
(37.7 \%)\end{array}$ & $\begin{array}{c}\text { Good Knowledge } \\
{[\mathrm{N}=\mathbf{2 5 0 ]}} \\
(\mathbf{6 2 . 3 \% )}\end{array}$ & & \\
\hline \multicolumn{5}{|l|}{ Age group } \\
\hline$<20$ & $8(5.3)$ & $12(4.8)$ & & \\
\hline $20-29$ & $81(53.6)$ & $135(54.0)$ & & \\
\hline $30-39$ & $54(35.8)$ & $85(34.0)$ & & \\
\hline $40-49$ & $7(4.6)$ & $17(6.8)$ & & \\
\hline$\geq 50$ & $1(0.7)$ & $1(0.4)$ & 0.99 & 0.910 \\
\hline \multicolumn{5}{|l|}{ Sex } \\
\hline Male & $3(2.0)$ & $2(0.8)$ & & \\
\hline Female & $148(98.0)$ & $248(99.2)$ & 1.08 & 0.299 \\
\hline \multicolumn{5}{|l|}{ Marital Status } \\
\hline Single & $30(19.9)$ & $49(19.6)$ & & \\
\hline Married & $89(58.9)$ & $152(60.8)$ & & \\
\hline Separated & $5(3.3)$ & $5(2.0)$ & & \\
\hline Divorced & $1(0.7)$ & $0(0.0)$ & & \\
\hline Cohabiting & $26(17.2)$ & $44(17.6)$ & 2.37 & 0.668 \\
\hline \multicolumn{5}{|l|}{ Educational Level } \\
\hline Never & $23(15.2)$ & $32(12.8)$ & & \\
\hline Basic Education & $109(72.2)$ & $173(69.2)$ & & \\
\hline SHS & $14(9.3)$ & $34(13.6)$ & & \\
\hline Tertiary & $5(3.3)$ & $11(4.4)$ & 2.28 & 0.517 \\
\hline \multicolumn{5}{|l|}{ Religion } \\
\hline Christian & $143(94.7)$ & 237(94.8) & & \\
\hline Muslim & $5(3.3)$ & $11(4.4)$ & & \\
\hline African Traditional Worship & $3(2.0)$ & $2(0.8)$ & 1.34 & 0.511 \\
\hline \multicolumn{5}{|l|}{ Ethnicity } \\
\hline Akan & $24(15.9)$ & $48(19.2)$ & & \\
\hline Ewe & $105(69.5)$ & $149(59.6)$ & & \\
\hline $\mathrm{Ga}$ & $4(2.7)$ & $3(1.2)$ & & \\
\hline Krobo & $13(8.6)$ & $23(9.2)$ & & \\
\hline Others & $5(3.3)$ & $27(10.8)$ & 9.83 & 0.043 \\
\hline \multicolumn{5}{|l|}{ Occupation } \\
\hline Self Employed & $73(48.3)$ & $145(58.0)$ & & \\
\hline Government Employee & $1(0.7)$ & $11(4.4)$ & & \\
\hline Private Employee & $9(6.0)$ & $7(2.8)$ & & \\
\hline Not Employed & $68(45.0)$ & $87(34.8)$ & 10.92 & 0.012 \\
\hline \multicolumn{5}{|l|}{ Income (GHC) } \\
\hline$<200$ & $43(63.2)$ & $80(56.7)$ & & \\
\hline $200-400$ & $13(19.1)$ & $39(27.7)$ & & \\
\hline $401-600$ & $7(10.3)$ & $9(6.4)$ & & \\
\hline $601-800$ & $4(5.9)$ & $5(3.6)$ & & \\
\hline$>800$ & $1(1.5)$ & $8(5.7)$ & 5.05 & 0.282 \\
\hline \multicolumn{5}{|l|}{ Age of Child (Months) } \\
\hline $0-11$ & $37(24.5)$ & $53(21.2)$ & & \\
\hline $12-23$ & $45(29.8)$ & $75(30.0)$ & & \\
\hline $24-59$ & $69(45.7)$ & $122(48.8)$ & 0.65 & 0.723 \\
\hline \multicolumn{5}{|l|}{ Sex of Child } \\
\hline Male & $72(47.7)$ & $99(39.6)$ & & \\
\hline Female & $79(52.3)$ & $151(60.4)$ & 2.51 & 0.113 \\
\hline
\end{tabular}




\begin{tabular}{lcccc}
\hline & \multicolumn{2}{c}{ Level of Knowledge } & & \\
\cline { 2 - 3 } Variable & $\begin{array}{c}\text { Poor Knowledge } \\
{[\mathbf{N}=\mathbf{1 5 1}]} \\
\mathbf{( 3 7 . 7 \% )}\end{array}$ & $\begin{array}{c}\text { Good Knowledge } \\
{[\mathbf{N}=\mathbf{2 5 0 ]}} \\
\mathbf{( 6 2 . 3 \% )}\end{array}$ & $\begin{array}{c}\text { Chi-square } \\
\boldsymbol{\chi}^{\mathbf{2}}\end{array}$ & p-value \\
\hline Place of Delivery & $131(86.7)$ & $196(78.4)$ & 4.37 & $\mathbf{0 . 0 3 7}$ \\
Health Facility & $20(13.3)$ & $54(21.6)$ & & \\
Home & & &
\end{tabular}

Table 4: Predictors of Good Knowledge of Mothers/Caregivers on Immunization

\begin{tabular}{|c|c|c|c|c|c|}
\hline Variable & $\begin{array}{c}\text { Good Knowledge } \\
{[\mathbf{N}=\mathbf{2 5 0}]} \\
(\mathbf{6 2 . 3 \% )}\end{array}$ & $\operatorname{COR}(95 \% \mathrm{CI})$ & p-value & AOR (95\%) & $\begin{array}{c}\text { p- } \\
\text { value }\end{array}$ \\
\hline \multicolumn{6}{|l|}{ Age group } \\
\hline$<20$ & $12(4.8)$ & 1 & & & \\
\hline $20-29$ & $135(54.0)$ & $1.11(0.44-2.83)$ & 0.825 & & \\
\hline $30-39$ & $85(34.0)$ & $1.05(0.40-2.73)$ & 0.921 & & \\
\hline $40-49$ & $17(6.8)$ & $1.62(0.46-5.68)$ & 0.452 & & \\
\hline$\geq 50$ & $1(0.4)$ & $\begin{array}{c}0.67(0.04- \\
12.27)\end{array}$ & 0.785 & & \\
\hline \multicolumn{6}{|l|}{ Sex } \\
\hline Male & $2(0.8)$ & 1 & & & \\
\hline Female & $248(99.2)$ & $\begin{array}{l}2.51(0.42- \\
15.22)\end{array}$ & 0.316 & & \\
\hline \multicolumn{6}{|l|}{ Marital Status } \\
\hline Single & 49(19.6) & 1 & & & \\
\hline Married & $152(60.8)$ & $1.05(0.62-1.77)$ & 0.868 & & \\
\hline Separated & $5(2.0)$ & $0.61(0.16-2.29)$ & 0.466 & & \\
\hline Divorced & $0(0.0)$ & & & & \\
\hline Cohabiting & $44(17.6)$ & $1.04(0.53-2.01)$ & 0.917 & & \\
\hline \multicolumn{6}{|l|}{ Educational Level } \\
\hline Never & $32(12.8)$ & 1 & & & \\
\hline Basic Education & $173(69.2)$ & $1.14(0.63-2.05)$ & 0.660 & & \\
\hline SHS & $34(13.6)$ & $1.75(0.77-3.97)$ & 0.184 & & \\
\hline Tertiary & $11(4.4)$ & $1.58(0.48-5.17)$ & 0.449 & & \\
\hline \multicolumn{6}{|l|}{ Religion } \\
\hline Christian & $237(94.8)$ & & & & \\
\hline Muslim & $11(4.4)$ & $1.33(0.45-3.90)$ & 0.606 & & \\
\hline Traditional & $2(0.8)$ & $0.40(0.07-2.44)$ & 0.322 & & \\
\hline \multicolumn{6}{|l|}{ Worship } \\
\hline \multicolumn{6}{|l|}{ Ethnicity } \\
\hline Akan & $48(19.2)$ & 1 & & & \\
\hline Ewe & $149(59.6)$ & $0.71(0.41-1.23)$ & 0.221 & $0.69(0.39-1.21)$ & 0.197 \\
\hline $\mathrm{Ga}$ & $3(1.2)$ & $0.38(0.08-1.81)$ & 0.222 & $0.40(0.08-1.99)$ & 0.266 \\
\hline Krobo & $23(9.2)$ & $0.88(0.38-2.05)$ & 0.774 & $0.92(0.39-2.17)$ & 0.844 \\
\hline Others & $27(10.8)$ & $2.70(0.92-7.89)$ & 0.070 & $2.78(0.93-8.29)$ & 0.066 \\
\hline \multicolumn{6}{|l|}{ Occupation } \\
\hline Self Employed & $145(58.0)$ & 1 & & & \\
\hline Government Employee & $11(4.4)$ & $\begin{array}{c}5.54(0.70- \\
43.73)\end{array}$ & 0.104 & $\begin{array}{c}5.28(0.66- \\
42.36)\end{array}$ & 0.117 \\
\hline Private Employee & $7(2.8)$ & $0.39(0.14-1.09)$ & 0.074 & $0.35(0.12-1.02)$ & 0.054 \\
\hline Not Employed & $87(34.8)$ & $0.64(0.42-0.98)$ & 0.042 & $0.62(0.40-0.95)$ & $\mathbf{0 . 0 3 0}$ \\
\hline \multicolumn{6}{|l|}{ Income (GHC) } \\
\hline$<200$ & $80(56.7)$ & 1 & & & \\
\hline $200-400$ & $39(27.7)$ & $1.61(0.78-3.34)$ & 0.199 & & \\
\hline $401-600$ & $9(6.4)$ & $0.69(0.24-1.98)$ & 0.492 & & \\
\hline $601-800$ & $5(3.6)$ & $0.67(0.17-2.63)$ & 0.568 & & \\
\hline$>800$ & $8(5.7)$ & $\begin{array}{c}4.30(0.52- \\
35.52)\end{array}$ & 0.176 & & \\
\hline
\end{tabular}




\begin{tabular}{|c|c|c|c|c|c|}
\hline Variable & $\begin{array}{c}\text { Good Knowledge } \\
{[\mathbf{N}=\mathbf{2 5 0}]} \\
(\mathbf{6 2 . 3 \% )}\end{array}$ & COR (95\% CI) & p-value & $\operatorname{AOR}(95 \%)$ & $\begin{array}{c}p- \\
\text { value }\end{array}$ \\
\hline \multicolumn{6}{|c|}{$\begin{array}{l}\text { Age of Child } \\
\text { (Months) }\end{array}$} \\
\hline $0-11$ & $53(21.2)$ & 1 & & & \\
\hline $12-23$ & $75(30.0)$ & $1.16(0.67-2.04)$ & 0.596 & & \\
\hline $24-59$ & $122(48.8)$ & $1.23(0.74-2.06)$ & 0.421 & & \\
\hline \multicolumn{6}{|l|}{ Sex of Child } \\
\hline Male & $99(39.6)$ & 1 & & & \\
\hline Female & $151(60.4)$ & $1.39(0.92-2.09)$ & 0.113 & & \\
\hline \multicolumn{6}{|c|}{ Place of Delivery } \\
\hline Health Facility & $196(78.4)$ & 1 & & & \\
\hline Home & $54(21.6)$ & $1.80(1.03-3.12)$ & 0.038 & $2.12(1.19-3.77)$ & 0.010 \\
\hline
\end{tabular}

Table 4 shows the association between the level of knowledge of mothers/caregivers and the demographic characteristics. Level of knowledge was significantly associated with ethnicity $\left(\chi^{2}=9.83, p=0.043, \alpha=0.05\right)$, occupation $\left(\chi^{2}=10.92, p=0.012, \alpha=0.05\right)$, and place of delivery $\left(\chi^{2}=4.37, p=0.037, \alpha=0.05\right)$. However, there was no association between level of knowledge and age, sex, marital status, educational level, religion, income level, age of child, and sex of child $(\mathrm{P}>0.05)$.

Table 5 shows the predictors of good knowledge among mothers/caregivers on immunization. Level of knowledge was significantly associated with ethnicity, occupation, and place of delivery. After adjusting for possible confounding effect of the variables, participants who were unemployed were $38 \%$ less likely to have good knowledge compared to participants who were self-employed and the difference was statistically significant $(\mathrm{AOR}=0.62 ; 95 \% \mathrm{CI}=0.40-0.95 ; \mathrm{p}=0.030$ ). Also, participants who delivered at home were 2.1 times more likely to have good knowledge compared to participants who delivered at the health facility $(\mathrm{AOR}=2.12$; $95 \% \mathrm{CI}=1.19-3.77 ; \mathrm{p}=0.010)$.

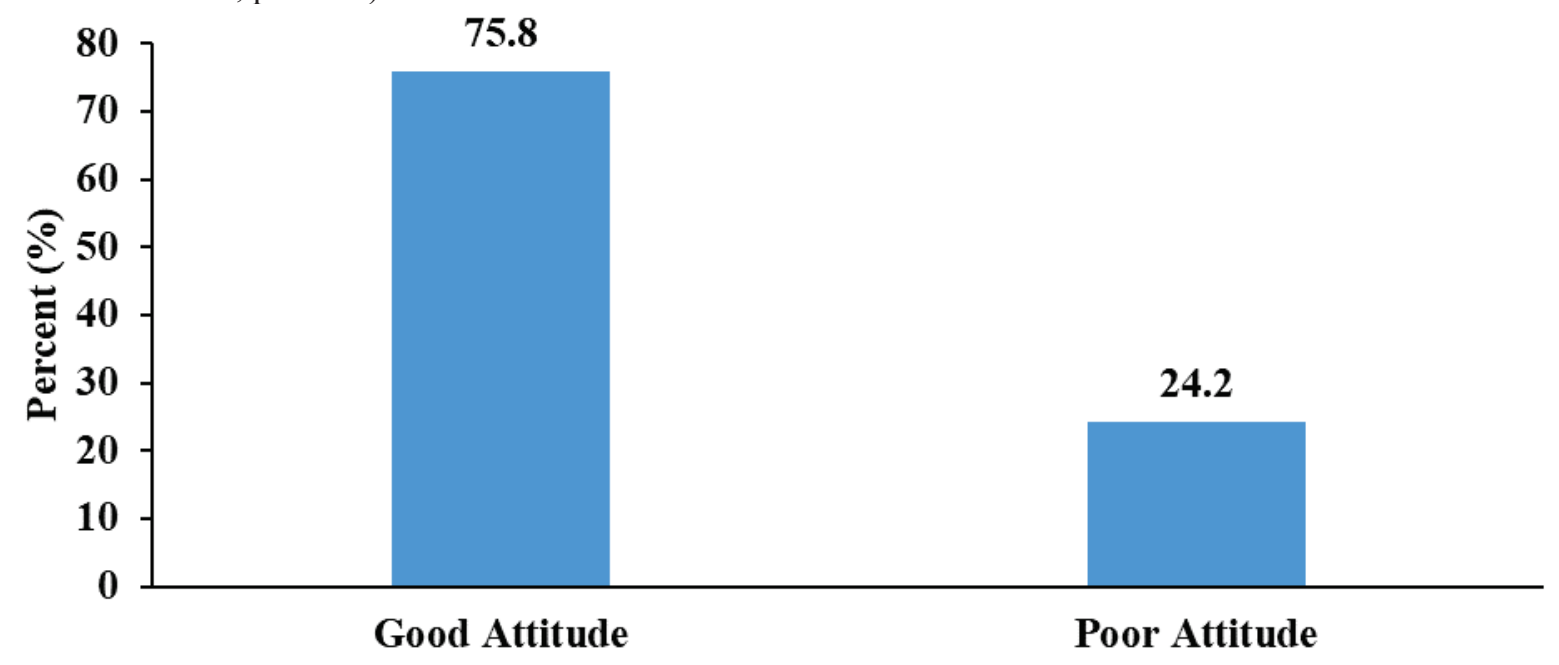

\section{Attitude}

Figure 4Attitude of Mothers/Caregivers Toward Immunization

Figure 4 shows the attitude of mothers/caregivers towards immunization. Overall, 304(75.8) of the participants had a good attitude toward immunization while $97(24.2 \%)$ had a poor attitude toward immunization. 
Table 5: Attitudes of Mothers/Caregivers Toward Immunization

\begin{tabular}{|c|c|c|}
\hline Variable & $\begin{array}{c}\text { Frequency } \\
{\left[\begin{array}{c}\mathrm{N}=401] \\
\text { (n) }\end{array}\right.}\end{array}$ & $\begin{array}{c}\text { Percent } \\
(\%)\end{array}$ \\
\hline \multicolumn{3}{|c|}{ Normally go for immunization services when the time is due } \\
\hline Yes & 368 & 91.8 \\
\hline No & 33 & 8.2 \\
\hline \multicolumn{3}{|c|}{ Waiting time at health facility } \\
\hline$<15$ mins & 182 & 45.4 \\
\hline $15-30$ mins & 146 & 36.4 \\
\hline $31-60 \mathrm{mins}$ & 47 & 11.7 \\
\hline$>1$ hour & 26 & 6.5 \\
\hline \multicolumn{3}{|c|}{ Vaccination session affect your schedules } \\
\hline Yes & 30 & 7.5 \\
\hline No & 371 & 92.5 \\
\hline \multicolumn{3}{|c|}{ Have means of transport to vaccination session } \\
\hline Yes & 351 & 87.5 \\
\hline No & 50 & 12.5 \\
\hline
\end{tabular}

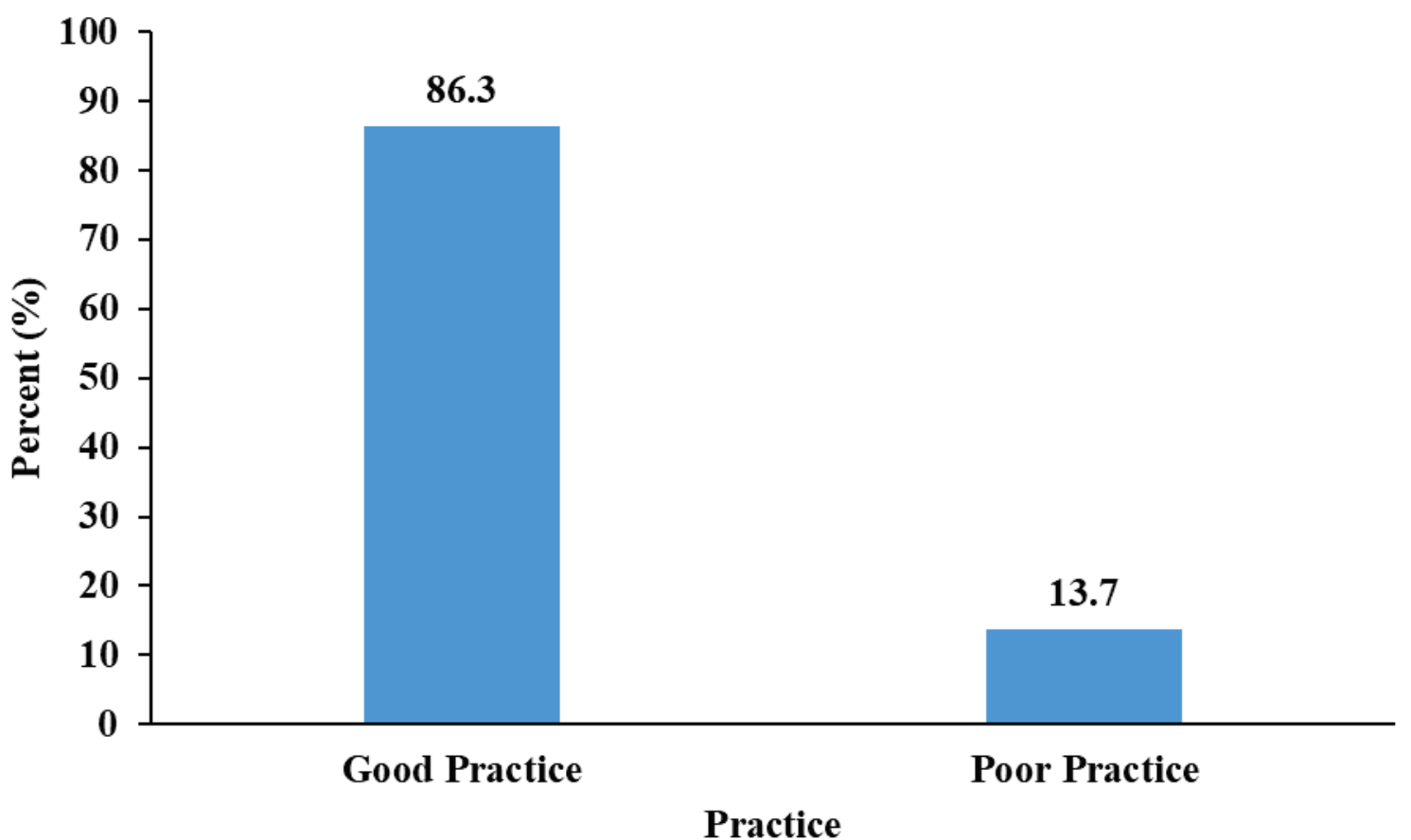

Figure 5 Practice of Immunization by Mothers/Caregivers

Figure 5 shows the practices of immunization by mothers/caregivers. Overall, 346(86.3\%) of the participants had good practices of immunization while $55(13.7 \%$ ) had poor practices of immunization. 
Table 6: Practices of Mothers/Caregivers Toward Immunization

\begin{tabular}{|c|c|c|}
\hline Variable & $\begin{array}{c}\text { Frequency } \\
{\left[\begin{array}{c}\mathrm{N}=401] \\
(\mathrm{n})\end{array}\right.}\end{array}$ & $\begin{array}{c}\text { Percent } \\
(\%)\end{array}$ \\
\hline \multicolumn{3}{|c|}{ Follow vaccination programs } \\
\hline Yes & 399 & 99.5 \\
\hline No & 2 & 0.5 \\
\hline \multicolumn{3}{|c|}{ Will search for other vaccines for children } \\
\hline Yes & 399 & 99.5 \\
\hline No & 2 & 0.5 \\
\hline \multicolumn{3}{|c|}{ Manage swelling after vaccination by cold compress } \\
\hline Yes & 396 & 98.7 \\
\hline No & 5 & 1.3 \\
\hline \multicolumn{3}{|c|}{ Use analgesics for swelling and pain after vaccination } \\
\hline Yes & 387 & 96.5 \\
\hline No & 14 & 3.5 \\
\hline \multicolumn{3}{|c|}{ I cancel some schedules to attend immunization sessions } \\
\hline Yes & 356 & 88.8 \\
\hline No & 45 & 11.2 \\
\hline
\end{tabular}

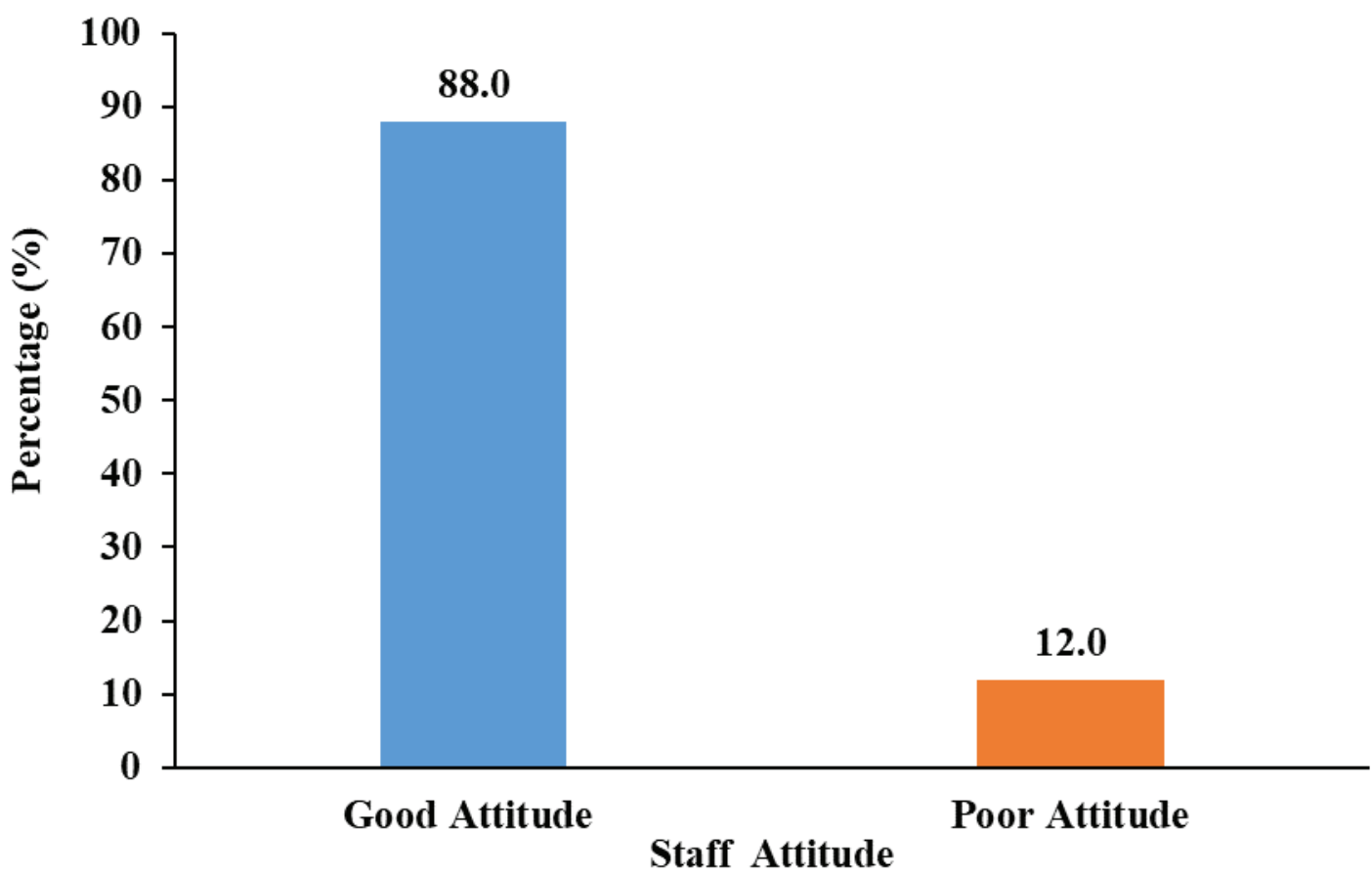

Figure 6 Staff Attitude towards Mothers/Caregivers During Immunization

Figure 6 shows the attitude of staff towards mothers/caregivers during immunization. Out of the 401 participants enrolled in the study, 353(88.0\%) reported a good staff attitude towards them while $48(12.0 \%)$ reported poor staff attitude. 
Table 7: Attitude of Health Staff towards Mothers/Caregivers during Immunization

\begin{tabular}{|c|c|c|}
\hline Variable & $\begin{array}{c}\text { Frequency } \\
{[\mathbf{N}=401]} \\
(\mathrm{n})\end{array}$ & $\begin{array}{c}\text { Percent } \\
(\%)\end{array}$ \\
\hline \multicolumn{3}{|c|}{ Health staff maltreat you during vaccination session } \\
\hline Yes & 25 & 6.2 \\
\hline No & 376 & 93.8 \\
\hline \multicolumn{3}{|c|}{ Health staff collect money during vaccination session } \\
\hline Yes & 29 & 7.2 \\
\hline No & 372 & 92.8 \\
\hline \multicolumn{3}{|c|}{ Meet health staff at vaccination site for vaccination } \\
\hline Yes & 396 & 98.8 \\
\hline No & 5 & 1.2 \\
\hline \multicolumn{3}{|c|}{ Frequency of meeting health staff at vaccination site } \\
\hline Very Often & 365 & 91.0 \\
\hline Sometimes & 31 & 7.7 \\
\hline Not Often & 5 & 1.3 \\
\hline \multicolumn{3}{|c|}{ Health staff have counseling during child welfare clinic } \\
\hline Yes & 383 & 95.5 \\
\hline No & 18 & 4.5 \\
\hline \multicolumn{3}{|c|}{ Health staff scream at you when you forget your child's card } \\
\hline Yes & 28 & 7.0 \\
\hline No & 373 & 93.0 \\
\hline \multicolumn{3}{|c|}{$\begin{array}{l}\text { Health staff scream at you when you when you miss a schedule for } \\
\text { vaccination }\end{array}$} \\
\hline Yes & 35 & 8.7 \\
\hline No & 366 & 91.3 \\
\hline \multicolumn{3}{|c|}{ Health staff scream at you when you have a malnourished child } \\
\hline Yes & 58 & 14.5 \\
\hline No & 343 & 85.5 \\
\hline \multicolumn{3}{|c|}{$\begin{array}{l}\text { Health staff scream at you when you are poorly dressed or had dirty } \\
\text { clothing }\end{array}$} \\
\hline Yes & 18 & 4.5 \\
\hline No & 383 & 95.5 \\
\hline
\end{tabular}

From table 8 , a total of $25(6.2 \%)$ participants reported that health staff maltreated them during vaccination session, with $29(7.2 \%)$ of them claiming that the health staff collected money from them. Majority of the caregivers, 396(98.8\%) reported meeting health staff at vaccination site for vaccination. Also, 383(95.5\%) participants reported having a counseling session during CWC. Various proportion of the participants reported screaming on them when they forget their child's card $28(7.0 \%)$, when they miss an immunization session $35(8.7 \%)$, when their child become malnourished 58(14.5\%) and when they are poorly dressed $18(4.5 \%)$.

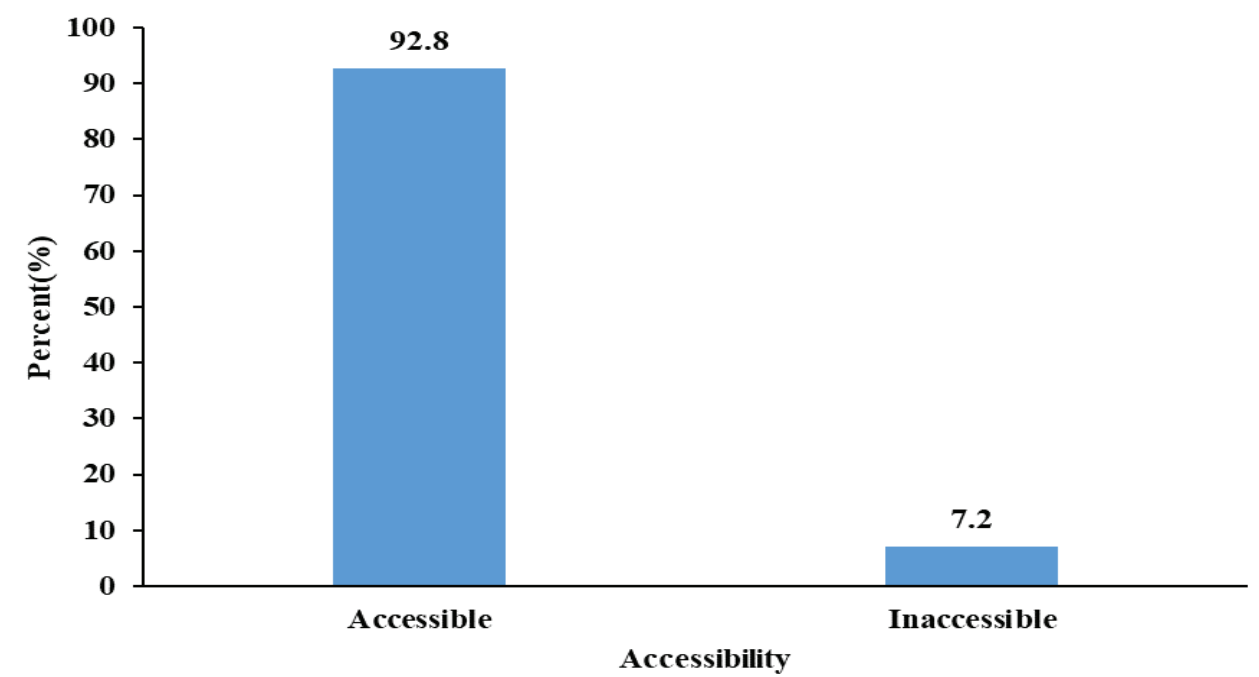

Figure 7 Accessibility of Immunization Services 
Figure 7 shows the accessibility of immunization services to mothers/caregivers. Majority 372(92.8\%) of the mothers/caregivers had accessible immunization services.

From table 9, majority of the participants, 382(95.3\%) reported having vaccination site close to their place of residence. Most of the caregivers, 284(70.8\%) travelled less than 15 minutes to get to their vaccination centers and $99(24.7 \%)$ had to travel between 15-30 minutes to get to their vaccination centers.

Table 8: Accessibility of Immunization Services

\begin{tabular}{lcc}
\hline Variable & Frequency [N=401] & $\begin{array}{c}\text { Percent } \\
(\%)\end{array}$ \\
\hline Any accessible vaccination site to your residing area & & 95.3 \\
Yes & 382 & 4.7 \\
No & 19 & 70.8 \\
Travel time to nearest vaccination site & & 24.7 \\
$<15$ mins & 284 & 4.0 \\
$15-30$ mins & 99 & 0.5 \\
$31-60$ mins & 16 & 2 \\
$>1$ hour & & \\
\hline
\end{tabular}

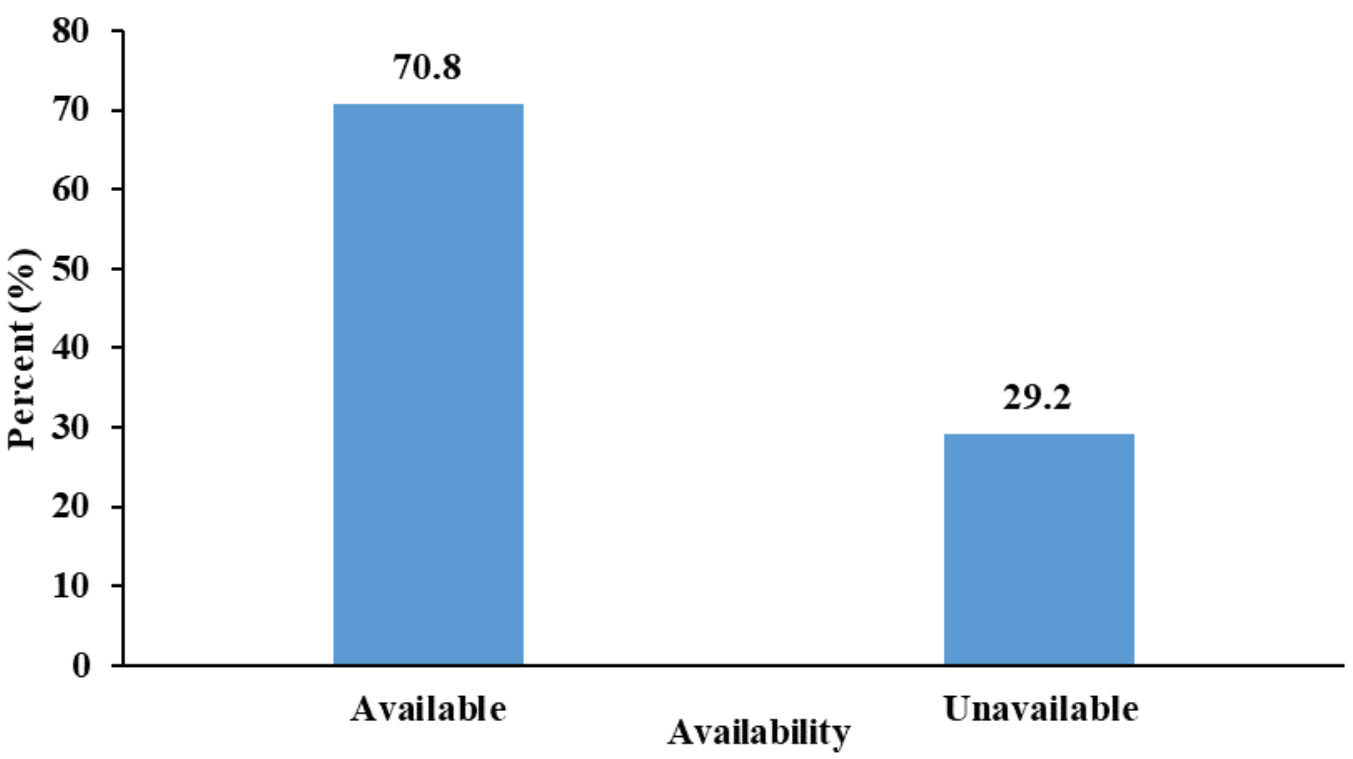

Figure 8 Availability of Immunization Services

Figure 8 shows the availability of immunization services. A total of $284(70.8 \%)$ mothers/caregivers reported having available immunization services in their community.

From table 10, most of the caregivers $284(70.8 \%)$ knew their local vaccination site schedule and $382(95.3 \%)$ claimed they were conveniently located. Most of caregivers $283(70.6 \%)$ said the vaccination service schedules were never cancelled and 314(78.3\%) claimed they never returned from vaccination session without getting their children vaccinated.

Table 9: Availability of Immunization Services

\begin{tabular}{lcc}
\hline Variable & $\begin{array}{c}\text { Frequency [N=401] } \\
\text { (n) }\end{array}$ & $\begin{array}{c}\text { Percent } \\
\text { (\%) }\end{array}$ \\
\hline Know your local vaccination site schedule & 284 & 70.8 \\
Yes & 117 & 29.2 \\
No & & 95.3 \\
Convenient with the vaccination services provided & 382 & 4.7 \\
Yes & 19 & 29.4 \\
No & & 70.6 \\
Any cancellation of vaccination schedule & 118 & 21.7 \\
Yes & 283 & 78.3 \\
No & & 87 \\
Yes returned without getting vaccinated & 314 & \\
No & & \\
\hline
\end{tabular}




\section{Discussion}

\section{Knowledge, Attitude, and Practices of Mothers/Caregivers towards Immunization}

This study revealed that mothers/caregivers attending immunization session in the Asuogyaman district had a fairly good knowledge on immunization (62.3\%). The high level of knowledge is important to make mothers/caregivers take their children to immunization sessions since they know its advantage to the children. The level of knowledge found in this study is however higher than what was found by Birhanu et al (2016) in Ethiopia. In their study, they found that out of 626 mothers who were enrolled in the study, 55.0\% had good knowledge on childhood immunization. This difference could be attributed to the health talks delivered at the health facilities as majority of the mothers/caregivers in this study $(86.0 \%)$ mentioned their source of information to be from health workers compared to only $48.2 \%$ receiving information about immunization from health workers in the study in Ethiopia. Hence there is a likelihood of them receiving more accurate information. The level of knowledge found in this study was however lower than what was found by Alenazi et al. (2017) in Egypt, Joseph, Devarashetty, Reddy, and Sushma (2015) in Bengaluru in India and Oryema et. al (2017) in Uganda. In their study, the overall knowledge were $87.2 \%, 84 \%$ and $97 \%$ respectively. This discrepancy could be attributed to the higher educational level among the mothers in the study by Alenazi et al. as majority of their participants had either secondary school $(33.8 \%)$ or college $(61 \%)$ education with only $5.2 \%$ having primary school education. This study found that mothers/caregivers who were unemployed were less likely to have good knowledge compared to those who were self-employed. This could be due to the inability of mothers who were unemployed to transport themselves to enable them access healthcare services due to financial problems, hence depriving them of the privilege to get knowledge on immunization services. Also, mothers who delivered at the home were more likely to have good knowledge compared to mothers who delivered at the health facility. This could be due to the fact that the district under study is made up of many rural communities, and though the mothers may attend antenatal clinic during their pregnancy, most may prefer to deliver at home due to their individual preferences or societal influence. This assumption is however subject to further investigation.

This study found the overall attitude of mothers/caregivers toward immunization services to be positive. From this study, about 8 out of every 10 mother/caregiver had a positive attitude towards immunization. This implies that most of the mothers/caregivers enrolled in the study have placed a high value on immunization services and do everything possible to make sure their children get vaccinated. This positive attitude toward immunization would help in increasing the immunization coverage and also ensure that children are vaccinated against infectious diseases thereby giving herd immunity to other children who do not get vaccinated. However, a study conducted by Birhanu, Anteneh, Kibie, \& Jejaw (2016) in Ethiopia found only 53.8\% of mothers having a positive attitude towards immunization. This discrepancy could be due to the larger sample used in the study by Birhanu et al in Ethiopia (Birhanu et al., 2016). This study also found that about $92 \%$ of the mothers/caregivers normally went for immunization sessions. This shows that the mothers place a high value on immunization as they make sure they go for immunization sessions when the time is due. Most of the mothers/caregivers waited less than 30 minutes before they were attended to by health staff. With the waiting time not being long, mothers are likely to go for immunization sessions since their schedules for the day will not be affected that much due to waiting at the health facility for long. Also, immunization sessions did not affect the schedules of about $93 \%$ of the mothers/caregivers and this could be attributed to the short waiting time at the health facility. About $88 \%$ of the mothers/caregivers also had a means of transport to the immunization centers and this could also account for the high positive attitude exhibited by mothers/caregivers toward immunization. With these factors being in place, the attitude of mothers toward immunization is likely to be high as ChrisOtubor et al. (2015) identified busy schedules of mothers, long waiting time, and transportation challenges to be contributing factors to low immunization coverage (Chris-Otubor et al., 2015).

Mothers/Caregivers practices toward immunization was generally high in this study. About 9 out of every 10 mothers/caregivers had good practices toward immunization activities. Similarly, Birhanu et al. (2016) in their study in Ethiopia found that $84 \%$ of the respondents had good practices toward immunization (Birhanu et al., 2016). This implies that most mothers/caregivers enrolled in the study carry out activities that promote or enhance immunization activities and this can contribute positively to increasing immunization levels. In this study, 98.7\% reported they manage swelling after vaccination by cold compress and $96.5 \%$ said they used analgesics for swelling pain after vaccination. This practice could be due to the counseling given mothers/caregivers during immunization on how to manage any adverse reaction to immunization. This confirms what was found by Alenazi et al. (2017) in their study in Italy who recorded that $85.2 \%$ managed swelling by cold compress, and $87.2 \%$ used analgesics for swelling and pain after vaccination (Alenazi et al., 2017). However, a survey conducted in Uganda argued that fear of side effects, ignorance, laziness, of mothers/caregivers contribute to low immunization patronage (Vonasek et al., 2016). Also, a study conducted in Techiman Municipality in Ghana reported that low immunization coverage was attributed to mothers being too busy with other tasks and inconvenient time of immunization schedules (Adokiya, Baguune, \& Ndago, 2017). 


\section{Attitude of Health Staff Towards Immunization}

In this study, $88 \%$ of the mothers/caregivers reported that health staff exhibited a good attitude towards them. Similarly, Amin et al. (2013) in their study in urban Dili, Timor-Leste reported $97 \%$ of mothers expressing satisfaction with the services given them and the health workers' attitude towards mothers appeared to be friendly (Amin et al., 2013). On the other hand, a study revealed that $49 \%$ of the respondents reported bad attitude of health workers toward them (Favin et al., 2012). This difference can be attributed to the different study design employed in the study by Favin et al. as their study was a review of grey literature. However, less than $10.0 \%$ of the mothers/caregivers reported health staff exhibiting poor attitude. An exception of $15.0 \%$ of the caregivers complained that health staff screamed at them when their children became malnourished. This study also found that $98.8 \%$ of the mothers/caregivers reported meeting health staff at the vaccination centers and only $7.2 \%$ reported that health staff collect money from them at vaccination centers. This is also contrary to what was reported by Favin et al. (2012), who reported that some health workers maltreated the mothers/caregivers by illicitly charging them for vaccination and some also arriving late to start immunization sessions.

\section{Accessibility and Availability of Immunization Services}

Accessibility to health facilities that provide immunization services and availability of immunization services are some of the factors that have been identified to have an impact on immunization. This study found that $92.8 \%$ of the mothers/caregivers had access to health facilities that provide immunization services. This implies that the health facilities that provide immunization services are close to the respondents hence most of the mothers/caregivers have ready access to immunization services. Similarly, Legesse and Dechasa in their study in Ethiopia reported $98.8 \%$ of the respondents reported having access to health facilities that provide immunization services (Legesse \& Dechasa, 2015). On the other hand, a similar study conducted by Meleko, Geremew, \& Birhanu (2017) in Southwest Ethiopia recorded 56.5\% of the mothers/caregivers reporting having poor access to health facilities that provide immunization by foot (Meleko, Geremew, \& Birhanu, 2017). Also long walking distance from home to nearest facility and lack of transportation making it difficult to access health facility were associated factors to low immunization coverage (Jani, De Schacht, Jani, \& Bjune, 2008). This discrepancy can be attributed to the means of transport specified in the study by Meleko, Geremew, \& Birhanu and Jani, De Schacht, Jani, \& Bjune In their study, accessibility was focused on transport by foot alone while in the current study, accessibility was generalized to any means of transport. In this study, majority of the participants had to travel less than thirty minutes to get to the immunization centers (Meleko, Geremew, \& Birhanu, 2017). This implies most of the mothers/caregivers did not have to travel for long before getting access to immunization services.

Availability of immunization services is also another factor identified by researchers as contributing to low immunization coverage. This study found that $70.8 \%$ of mothers/caregivers reported they had available immunization services when they need it. In this study, $21.7 \%$ of the mothers/caregivers reported ever returning home without their children getting vaccinated. Further research is however needed to identify the specific reasons for which they had to return home without their children getting vaccinated. Similarly, Legesse \& Dechasa (2015) recorded $20.7 \%$ of mothers reporting they had to return back home without their children getting vaccinated, and this was due to unavailability of health providers to provide services and lack of vaccines at the facilities (Legesse \& Dechasa, 2015).

\section{STUDY LIMITATION}

This study did not include all the communities in the district due to limited resources. The study did not determine the immunization status of the children.

\section{CONCLUSIONS}

Mothers/caregivers in Asuogyaman district attending immunization sessions had a fairly good knowledge on immunization with positive attitude and good practices toward immunization activities. In addition, majority of the mothers/caregivers reported that health staff exhibited a positive attitude towards them. Mothers' occupation (not employed), ethnicity and place of delivery were the major factors determining immunization status of children.

\section{RECOMMENDATIONS}

Health education activities on immunization should be intensified by the community health nurses to increase the mothers/caregivers' knowledge on immunization in order to help increase the immunization coverage in the district. Also, outreach points should be created in every community through a collaboration between the district health management team and the local authorities to reduce the long waiting time and travel time of mothers to outreach point. Mothers should be encouraged by the community health workers and midwives to deliver at health facility, however those who delivers at home should be advised to visit health facility after delivery to 
immediately begins with immunization. In-service training should periodically be organized by the district health management team for the health workers to maintain the good practices and to improve upon their knowledge in immunization in the District. Finally, there should be a social support groups created in the community by the community health workers with support from women leaders in the community to empower mothers/caregivers to improve on their knowledge on immunization.

\author{
LIST OF ABBREVIATIONS AND ACRONYM \\ BCG: $\quad$ BACILLUS CALMETTE GUERIN \\ CWC: $\quad$ CHILD WELFARE CLINIC \\ DHD: $\quad$ DISTRICT HEALTH DIRECTORATE \\ DHMT: $\quad$ DISTRICT HEALTH MANAGEMENT TEAM \\ DPT: $\quad$ DIPHTHERIA-PERTUSSIS-TETANUS \\ EPI: $\quad$ EXPANDED PROGRAMME ON IMMUNIZATION \\ GHS: GHANA HEALTH SERVICE \\ GVAP: GLOBAL VACCINE ACTION PLAN \\ HIB: HAEMOPHILUS INFLUENZA TYPE B \\ MEN A: $\quad$ MENINGITIS \\ MOH: $\quad$ MINISTRY OF HEALTH \\ MR: $\quad$ MEASLES RUBELLA \\ OPV: $\quad$ ORAL POLIO VACCINE \\ PCV: $\quad$ PNEUMOCOCCAL CONJUGATE VACCINE \\ TT: $\quad$ TETANUS TOXOID \\ UNICEF: $\quad$ UNITED NATIONS CHILDREN'S EMERGENCY FUND \\ VPDs: $\quad$ VACCINE PREVENTABLE DISEASES \\ WHO: WORLD HEALTH ORGANIZATION \\ CHPS COMMUNITY-BASED HEALTH PLANNING AND SERVICES
}

\title{
DECLARATIONS
}

\section{ETHICS APPROVAL AND CONSENT TO PARTICIPATE}

Ethical approval was obtained from the University of Health and Allied Sciences Ethics Review Committee (UHAS-ERC) with reference number UHAS-REC A4 [95] 18-19.

\section{CONSENT FOR PUBLICATION}

Not applicable

\section{AVAILABILITY OF DATA AND MATERIAL}

All data generated or analyzed during this study are available with the corresponding author on reasonable request.

\section{COMPETING INTERESTS}

We declare that we have no conflict of interests

FUNDING:

The study was funded by the Principal Investigator

\section{AUTHORS' CONTRIBUTIONS}

SAB, ESK and AZA conceived the study. CAK and SAB did the data analysis. SAB, JMG, ESK were responsible for the initial draft of the manuscript. All authors reviewed and approved the final version of the manuscript.

\section{ACKNOWLEDGEMENTS}

We acknowledge all the entire staff of Asuogyaman District Health Directorate especially the Disease control officers for their unflinching support and the hard working data collectors and study respondents who participated in the interviews.

\section{REFERENCES}

Adokiya, M. N., Baguune, B., \& Ndago, J. A. (2017). Evaluation of immunization coverage and its associated factors among children 12-23 months of age in Techiman Municipality, Ghana, 2016. Archives of Public Health, 75(1), 28.

Alenazi, A.A.S., Alshareef, R.A., Alabudib, F.A., Alsayagh, Alsayagh, Y.M., \& Almuqarrab, A.J.H. (2017). Assessment of Knowledge and Attitude and Practice of Parents about Immunization in Jeddah City, 2017. The Egyptian Journal of Hospital Medicine, 69(7), 2939-2943.

Amin, R., de Oliviera, T.J.C., Da Cunha, M., Brown, T.W., Favin, M., \& Cappelier, K. (2013). Factors limiting immunization coverage in urban Dili, Timor-Leste. Global Health Science And Practice. 1(3):417-427

Asuogyaman District Health Directorate (2017). Annual district health report. 
Asuogyaman District Health Directorate (2017). District health profile

Birhanu, S., Anteneh, A., Kibie, Y., \& Jejaw, A. (2016). Knowledge, Attitude and Practice of Mothers Towards Immunization of Infants in Health Centres at Addis Ababa, Ethiopia. Am J Health Res, 4(1), 6-17.

Chris-Otubor, G. O., Dangiwa, D. A., Ior, L. D., \& Anukam, N. C. (2015). Assessment of knowledge, attitudes and practices of mothers in Jos North regarding immunization. IOSR Journal of Pharmacy, 5(6), 34-45.

Favin, M., Steinglass, R., Fields, R., Banerjee, K., \& Sawhney, M. (2012). Why children are not vaccinated: a review of the grey literature. International health, 4(4), 229-238.

Ghana Statistical Service (GSS), Ghana Health Service (GHS), and ICF International. 2015. Ghana Demographic and Health Survey 2014. Rockville, Maryland, USA: GSS, GHS, and ICF International.

Jani, J. V., De Schacht, C., Jani, I. V., \& Bjune, G. (2008). Risk factors for incomplete vaccination and missed opportunity for immunization in rural Mozambique. BMC public health, 8(1), 161.

Legesse, E., \& Dechasa, W. (2015). An assessment of child immunization coverage and its determinants in Sinana District, Southeast Ethiopia. BMC pediatrics, 15(1), 31.

Meleko, A., Geremew, M., \& Birhanu, F. (2017). Assessment of Child Immunization Coverage and Associated Factors with Full Vaccination among Children Aged 12-23 Months at Mizan Aman Town, Bench Maji Zone, Southwest Ethiopia. International Journal of Pediatrics, 2017.

Mihigo, R., Okeibunor, J., Anya, B., Mkanda, P., \& Zawaira, F. (2017). Challenges of immunization in the African Region. The Pan African medical journal, 27(Suppl 3).

MOH/GHS (2016). National Policy Guidelines on immunization in Ghana. Accra, Ghana.

Oryema, P., Babirye, J. N., Baguma, C., Wasswa, P., \& Guwatudde, D. (2017). Utilization of outreach immunization services among children in Hoima District, Uganda: a cluster survey. BMC research notes, 10(1), 111.

Vonasek, B. J., Bajunirwe, F., Jacobson, L. E., Twesigye, L., Dahm, J., Grant, M. J., ... \& Conway, J. H. (2016). Do maternal knowledge and attitudes towards childhood immunizations in rural Uganda correlate with complete childhood vaccination? PloS one, 11(2), e0150131.

WHO/UNICEF. (2017). WHO 1 in 10 infants worldwide did not receive any vaccinations in 2016. Who, 20162018. Retrieved from http://www.who.int/mediacentre/news/releases/2017/infants-worldwidevaccinations/en/?utm source

World Health Organization. (2015). WHO Immunization. Retrieved from http://www.who.int/topics/immunization/en/

World Health Organization. (2016a). immunization in Africa. Retrieved from https://doi.org/10.1017/CBO9781107415324.004

World Health Organization. (2018). Media centre Immunization coverage, 1-5.

World Health Organization. Updated data on immunization coverage published by WHO and UNICEF; 2015. Retrieved from http://www.who.int/topics/immunization/en/ 